\title{
El primer caso de feocromocitoma
}

\author{
Alejandro Román-Gonzáléz', Carlos Alfonso Builes-Barrera ${ }^{2}$, Johnayro Gutiérrez $z^{3}$
}

${ }^{1}$ Médico Internista y Endocrinólogo. Universidad de Antioquia Hospital Universitario San Vicente Fundación.

${ }^{2}$ Médico especialista en Endocrinología. Endocrinólogo de Adultos Hospital Universitario San Vicente Fundación. Docente de la sección de Endocrinología de la Universidad de Antioquia. Miembro de Número Asociación Colombiana de Endocrinología, Diabetes y Metabolismo.

${ }^{3}$ Médico Internista y Endocrinólogo. Universidad de Antioquia. Hospital Pablo Tobón Uribe, Docente Servicio de Endocrinología y Metabolismo, Universidad de Antioquia. Medellín (Colombia). Correspondencia:

Dr. Alejandro Román-González

Dirección electrónica: alejoroman@gmail.com

Fecha de recepción: 13/01/2016

Fecha de aceptación: 1/02/2016

\section{Resumen}

El feocromocitoma y el paraganglioma son tumores neuroendocrinos derivados de las células cromafines. El feocromocitoma está ubicado en la médula suprarrenal y el paraganglioma en los paraganglios. Esta enfermedad tiene un componente genético importante. Se considera infrecuente. En este artículo se presenta la historia del primer paciente reportado con feocromocitoma. Se trata de una mujer de 18 años alemana con palpitaciones, taquicardia, retinopatía hipertensiva, constipación y crisis de pánico que falleció en el Hospital de Friburgo de Brisgovia, en Alemania. La autopsia demostró la presencia de tumores adrenales bilaterales, 120 años después se confirmó la presencia de mutaciones en el proto-oncogen RET y los familiares de la paciente tenían feocromocitoma y carcinoma medular de tiroides. El primer caso conocido de feocromocitoma fue bilateral y secundario a una neoplasia endocrina múltiple tipo 2 .

\section{Palabras clave}

Feocromocitoma, paraganglioma, metanefrinas, neoplasia endocrina múltiple.

\begin{abstract}
Pheochromocytoma and paraganglioma are neuroendocrine tumors arising from chromafin cells. Pheochromocytoma is located in the adrenal medulla and paraganglioma are located in the paraganglioma. This disease has a strong genetic component. It is a very rare disease. In this manuscript, the history
\end{abstract}

of the first known report of pheochromocytoma is presented. An 18-year-old woman from Germany presents with palpitations, tachycardia, hypertensive retinopathy, constipation and panic attacks. The patient died at the Freiburg Hospital in Germany. The autopsy showed the presence of bilateral adrenal tumor. A mutation in the proto-oncogen RET was demonstrated 120 years later. Relatives of the patient had pheochromocytoma and medullary thyroid carcinoma. The first known case of pheochromocytoma had bilateral disease secondary to multiple endocrine neoplasia type 2.

\section{Key words}

Pheochromocytoma, paraganglioma, metanephrines, multiple endocrine neoplasia.

\section{Introducción}

El feocromocitoma es un tumor derivado de las células cromafines neuroendocrinas de la médula suprarrenal ${ }^{(1)}$ y como tal es capaz de producir no solamente catecolaminas sino otras hormonas como ACTH o PTHrp. El paraganglioma es un tumor similar pero localizado en los paraganglios ${ }^{(1)}$. El término previo feocromocitoma extra-adrenal no es correcto por las implicaciones clínicas que tiene la localización extraadrenal. En particular, el paraganglioma es un predictor clínico de metástasis, situación que no ocurre con el feocromocitoma ${ }^{(2)}$. Esta enfermedad es infrecuente y muchos clínicos no llegan nunca a conocer un paciente con este diagnóstico. En nuestro medio, la mayoría de publicaciones son reportes de caso $^{(3)}$. El feocromocitoma y el paraganglioma tienen una carga genética importante, tanto que hasta el $50 \%$ de ellos tienen un alteración genética somática o germinal que explica la génesis del mismo ${ }^{(4)}$.

El término feocromocitoma fue acuñado en 1912 por Ludwig Pick, un patólogo alemán, y proviene de las palabras griegas phaios ("oscura"), croma ("color") y cytoma ("tumor")"(5), Este término refleja la coloración oscura que toma este tumor cuando es expuesto a sales de ácido crómico. Sin embargo, este no fue el primer caso descrito de feocromocitoma. En el año 2006 se hizo honor al primer reporte conocido de esta enfermedad en el Primer Simposio Internacional de Feocromocitoma $^{(6)}$ y en este manuscrito se presenta la historia de este primer caso y el hallazgo de su etiología un siglo después. 


\section{Primer reporte conocido}

El primer caso de feocromocitoma fue descrito en 1886 por Felix Frankel originalmente en alemán ${ }^{(7)}$. Años después fue traducido al inglés y presentando como un Clásico en Oncología en 1984 en la revista CA: a Cancer Journal for Clinicians ${ }^{(8)}$. En el caso reportado, una paciente llamada Minna Roll, alemana, consultó al hospital de Friburgo (Freiburg, en alemán) de Brisgovia (figura 1). La paciente tenía 18 años y era previamente sana hasta los 17 años. Sus padres y seis hermanos eran sanos. En el invierno de 1883 inició con ataques repentinos de palpitaciones fuertes, seguidos por un fuerte vértigo y cefalea de pocos minutos de duración. Los episodios fueron más frecuentes y se asociaron con cefalea, vómito y, posteriormente, inició con constipación. Con el tiempo tuvo deterioro visual, cefaleas más intensas, vómito e incluso fiebre, todo esto obligó a que consultara al médico y requirió ser institucionalizada en el Hospital Universitario de Friburgo.

En el examen médico inicial se notó un pulso de 112 latidos por minuto, fuerte pero con la arteria un poco tensa y dura en la palpación. El examen oftalmológico demostró reti-

Figura 1. Mapa de Alemania con la ubicación de Friburgo

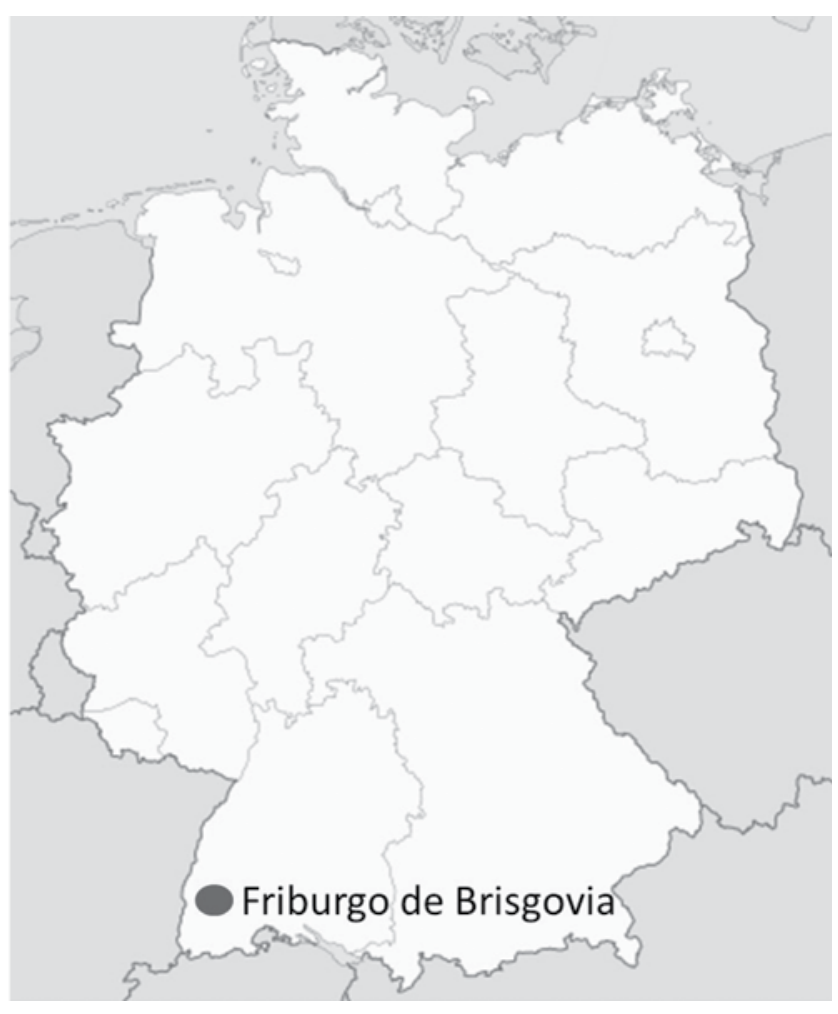

Tomado de Wikipedia con permiso*.

*(Permission is granted to copy, distribute and/or modify this document under the terms of the GNU Free Documentation License, Version 1.2 or any later version published by the Free Software Foundation; with no Invariant Sections, no Front-Cover Texts, and no Back-Cover Texts. A copy of the license is included in the section entitled GNU Free Documentation License.) nitis albuminúrica, hemorragias, exudados y papiledema consistente con una retinopatía hipertensiva grado IV. La evaluación cardiaca fue positiva para una pulsación epigástrica. La palpación tiroidea demostró un bocio considerable. Presentó varios episodios de palpitaciones intensas, cefalea, palidez, debilidad generalizada y sudoración fría. Solo se podían obtener deposiciones mediante enemas. El pulso varió entre 112 y 180 latidos por minuto. Se trató en ese momento con digitalis, bromuro de potasio, éter y en una ocasión recibió pilocarpina que disparó una crisis de ansiedad, vómito, disnea y taquicardia. La paciente falleció 10 días después de estar hospitalizada. Se realizó una autopsia el 21 de diciembre de 1884 . Se encontraron tumores adrenales bilaterales diagnosticados como sarcoma y angiosarcoma. El del lado izquierdo del tamaño de un puño y en el lado derecho de una avellana. No mencionan los hallazgos en la autopsia de la glándula tiroides. En ese reporte el Dr. Felix Frankel asume que el tumor proviene de la médula adrenal y que la masa tumoral y sus posibles secreciones asociadas sea lo que explique varios de los cambios de esta paciente. Para esa época era mucho lo que se desconocía sobre las glándulas adrenales, dado que Addison apenas había dirigido la atención a ellas en 1855 y básicamente se conocía la insuficiencia adrenal pero no las enfermedades asociadas a hiperproducción hormonal, éstas eran a la fecha desconocidas. En la figura 2 se presenta un corte de una glándula suprarrenal con un feocromocitoma y la foto macroscópica de un caso del Hospital Universitario San Vicente Fundación.

\section{Discusión clínica del primer caso}

Los síntomas del feocromocitoma son secundarios a la hipersecreción de catecolaminas y su efecto en diferentes órganos. La triada de síntomas clásicos de esta enfermedad (cefalea, diaforesis y palpitaciones) tiene pobre sensibilidad y especificidad y solo se encuentra en $30 \%$ de los pacientes ${ }^{(9)}$. Entre los otros síntomas descritos están ansiedad, pánico, diaforesis, disnea, epigastralgia, dolor torácico, cefalea, náusea, vómito, palidez, palpitaciones, temblor y manos frías ${ }^{(10,11)}$. La hipertensión puede ser episódica, continua o una presión arterial normal. Los ataques catecolaminérgicos son episódicos con una duración de 15 a 20 minutos y pueden presentarse varias veces al día. Pueden iniciar con una sensación de calor en cara y en tórax que progresa a palpitaciones, cefalea, temblor, palidez y diaforesis. Las manos son frías, lo mismo que los pies y puede existir fenómeno de Raynaud. Otros signos y síntomas que pueden ocurrir incluyen visión borrosa, papiledema, pérdida de peso, poliuria y polidipsia ${ }^{(12)}$. Raramente se asocia con cardiomiopatía, atribuida a un exceso de catecolaminas, la cual es similar a la cardiomiopatía inducida por el estrés (Takotsubo $)^{(13-16)}$. Los pacientes pueden presentar edema pulmonar ${ }^{(17)}$. En la tabla 1 presentamos las indicaciones clínicas para solicitar un estudio de feocromocitoma. 
Figura 2. Paciente de 46 años sexo masculino quien presenta dolor abdominal. En estudios de imagen se encontró la presencia de una masa adrenal de 92 por 55,4 por $54 \mathrm{~mm}$. Las catecolaminas en orina demostraron una producción elevada de adrenalina (162,97 mcg/24 horas, valor de referencia 0-20). Las metanefrinas totales en orina también estaban elevadas $(2,71 \mathrm{mg} / 24$ horas, valor de referencia $0-1 \mathrm{mg} / 24$ horas). Se realizó adrenalectomía y se confirmó un feocromocitoma. La primera imagen corresponde a una tinción con hematoxilina-eosina con un aumento $100 \mathrm{x}$ donde se logra apreciar la cápsula adrenal y la presencia de una neoplasia en la médula. En la foto 2 se aprecia el corte macroscópico del tumor.

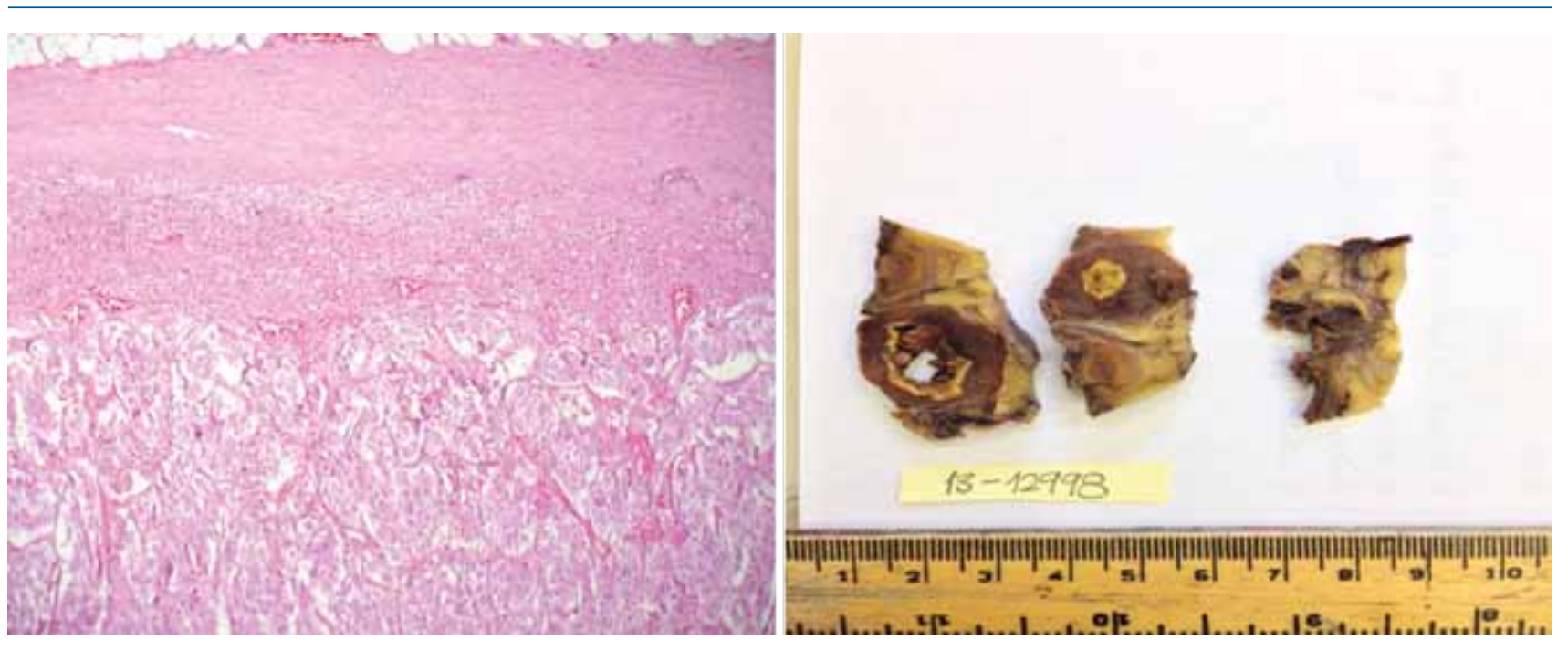

Imágenes Cortesía del Dr. Luis Fernando Arias. Departamento de Patología. Universidad de Antioquia.

En este caso, la paciente presentaba la mayoría de estas manifestaciones. No se menciona la presión arterial, dado que para esa época no se encontraba en uso clínico el tensiómetro, el cual se fue desarrollando en la segunda mitad del siglo XIX con los trabajos iniciales en 1855 por Vierordt y, posteriormente, por el desarrollo del esfigmomanómetro en 1881, por Samuel Siegfried Karl Ritter von Basch hasta la medición de la presión arterial como la conocemos hoy en día, que fue desarrollada en 1905 por el Dr. Nikolai Korotkoff ${ }^{(18)}$. Sin embargo, algunos de los cambios encontrados en la autopsia como hemorragias y edema pulmonar fueron atribuidos por el Dr. Felix Frankel al aumento súbito de la presión arterial durante los frecuentes episodios de vómito de la paciente.

Otro hallazgo interesante es la presencia de constipación. Este síntoma se presenta aproximadamente en el 6\% de los casos de feocromocitoma ${ }^{(11)}$. En este caso, la constipación de la paciente Minna Roll se puede clasificar como grado 2 con necesidad de uso regular de enemas. La constipación en estos pacientes mejora con el tratamiento del feocromocitoma pero usualmente requieren una dieta rica en fibra, uso de laxantes y estimulantes así como un consumo abundante de líquidos ${ }^{(11)}$.

Un hallazgo particular de la paciente es la edad de presentación. En niños, el feocromocitoma es raro ${ }^{(19)}$. Se estima que $20 \%$ de los casos ocurre en población pediátrica con un pico a los 11 años de edad y con predominio en varones. En pacientes menores de 18 años de edad se puede identificar una causa hereditaria en el $56 \%$ de los casos en estudios realizados antes
Tabla 1. Indicaciones para estudio de feocromocitoma

- Incidentaloma adrenal.

- Hipertensión arterial resistente.

- Falla cardiaca inexplicada.

- Hipertensión asociada a cefalea, palpitaciones, diaforesis y ataques de pánico.

- Crisis hipertensiva durante cirugía o anestesia general.

- Hipertensión inducida por beta-bloqueadores, inhibidores de la mono amino oxidasa, cambios en la micción o en la presión abdominal.

- Hipotensión ortostática en un paciente hipotenso.

- Diabetes de inicio reciente en un paciente joven y delgado con hipertensión.

- Enfermedades genéticas (Neoplasia endocrina múltiple tipo 2, Enfermedad de Von Hippel Lindau, neurofibromatosis tipo 1. Síndromes de paraganglioma familiar con mutaciones en la SDHB o SDHD).

- Familiares en primer grado con la enfermedad genética mencionada arriba.

del 2010. Posiblemente hoy en día este porcentaje sea mayor con los genes descubiertos en los últimos cinco años (vide infra). En menores de 10 años, las enfermedades hereditarias explican el $70 \%$ de los $\operatorname{casos}^{(19)}$. Por ende, es muy posible que 
este primer caso reportado de feocromocitoma tuviera una causa genética como bien se demostó años después.

\section{Hallazgos genéticos en el primer caso}

La combinación de la edad de la paciente y la presencia de un feocromocitoma bilateral es altamente sugestiva de un feocromocitoma hereditario. La presencia de una masa tiroidea sugiere la presencia de una neoplasia endocrina múltiple; sin embargo, en la autopsia de la paciente no reportan los hallazgos en tiroides. Ahora bien, también puede tratarse de una enfermedad de von Hippel Lindau o un síndrome de feocromocitoma paraganglioma familiar por mutaciones en la succinato deshidrogenasa. Todas estas hipótesis requieren confirmación genética.

Ciento veinte años después de este primer reporte, mediante una búsqueda en los familiares de la paciente, se logró determinar que se trataba de un feocromocitoma asociado a una neoplasia endocrina múltiple tipo 2 (MEN2) causada por una mutación en el proto-oncogen RET (Cis634Trp) ${ }^{(20)}$. Este interesante hallazgo fue presentado en The New England Journal of Medicine por investigadores de la Universidad de Friburgo en Alemania, el mismo sitio donde se hospitalizó la paciente un siglo antes. Este grupo de trabajo realizó una búsqueda de los familiares de la paciente y se pudo reconstruir el familiograma. Uno de los hermanos de Minna Roll tuvo tres hijos, dos varones y una niña, los tres tuvieron feocromocitoma. El segundo varón tuvo un hijo que desarrolló feocromocitoma y carcinoma medular de tiroides, lo mismo que su nieta. Todo el árbol genealógico puede observarse en la publicación inicial $^{(20)}$. Es de notar que estos investigadores pensaban que se trataba de una enfermedad de von Hippel-Lindau, dado que tenían casos ya conocidos de esta enfermedad en la región del Bosque Negro, de donde era originaria la paciente.

Hoy en día todos los pacientes con feocromocitoma deben tener la búsqueda de una causa genética ${ }^{(21)}$, especialmente desde la disponibilidad de secuenciación genética de siguiente generación que permite evaluar en una medición única la presencia de mutaciones en todos los genes descritos a la fechas asociados a feocromocitoma o paraganglioma

\section{Otros hitos históricos en la historia del feocromocitoma}

Aunque el Dr. Ludwig Pick fue quien acuñó el término feocromocitoma, en 1896 Manasse demostró que estos tumores se tornaban oscuros con las sales de cromo. El término paraganglioma fue descrito antes que el término feocromocitoma en 1908 por Alezais y Peyron para describir los tumores de los paraganglios ${ }^{(6)}$. La primera resección exitosa de un feocromocitoma fue realizada en 1926 en la Mayo Clinic en Estados Unidos por el Dr. C, H Mayo y por Roux en Suiza ${ }^{(6)}$.

Otro hito histórico que disparó una serie de estudios genéticos fue el hallazgo de mutaciones en la SHDD por Baysal y colaboradores $^{(22)}$. Este fenómeno describía por primera vez la asociación entre una alteración genética en una vía del metabolismo y una neoplasia. Posteriormente se fueron descubriendo mutaciones en otras subunidades de la SDH y mutaciones en otros genes como el TMEM127 por el grupo de la Dra. Patricia Dahia $^{(23)}$, en $\mathrm{MAX}^{(24,25)}$, en $\mathrm{FH}^{(26)}$, en EPAS1 ${ }^{(27)}$ y en el descubierto más reciente por el grupo de la Dra. Mercedes Robledo en el gen MDH2 que codifica para la enzima malato deshidrogena$\mathrm{sa}^{(28)}$. Esta pléyade de mutaciones genéticas hace que hoy en día el $50 \%$ de los feocromocitomas y paragangliomas sean causados por un evento genético (somático o germinal) ${ }^{(21)}$.

\section{Perspectivas futuras}

El feocromocitoma y los paragangliomas son enfermedades complejas y solo unos pocos centros tienen experiencia suficiente en estas entidades por su baja frecuencia de presentación. Actualmente, la Universidad de Antioquia se encuentra realizando un estudio sobre feocromocitoma en los dos hospitales universitarios de cuarto nivel de Medellín (Hospital Universitario San Vicente Fundación y Hospital Pablo Tobón Uribe) y se planea luego presentar los resultados a la comunidad médica y especialmente a los endocrinólogos e internistas de Colombia para conformar el registro nacional de esta enfermedad.

Por otro lado, un reto enorme en el manejo de esta enfermedad es el feocromocitoma maligno, definido por la presencia de metástasis ${ }^{(29)}$. En el MD Anderson, la experiencia del Dr. Camilo Jiménez, experto mundial en feocromocitoma maligno ${ }^{(1)}$ estima en un $17 \%$ la frecuencia de feocromocitomas malignos ${ }^{(2)}$.

La medicina basada en la evidencia dicta que el mejor nivel de evidencia proviene de metanálisis y los estudios clínicos controlados. En una enfermedad rara es difícil realizar estudios controlados con placebo, pero el esfuerzo realizado por el Dr. Camilo Jiménez en este sentido es descomunal y posiblemente cambie el paradigma de tratamiento del feocromocitoma y el paraganglioma maligno. Actualmente se encuentra realizando estudios para esta enfermedad con cabozantinib (Cabozantinib for Malignant Pheochromocytoma NCT02302833 clinicaltriasl. gov), un inhibidor de tirosina cinasa, para pacientes con feocromocitoma y paraganglioma maligno en progresión. El desenlace primario de este estudio es la tasa de respuesta total evaluada por Recist 1.1. El segundo estudio que se está realizando es con Azedra (A Study Evaluating Ultratrace Iobenguane I 131 (MIBG) in Patients With Malignant Pheochromocytoma/ Paraganglioma NCT00874614 clinicaltrials.gov) en pacientes con feocromocitoma maligno en recaída o refractarios a otra terapia que tengan positividad en un estudio nuclear con meta-yodo bencil guanidina. Los resultados preliminares fueron presentados en ENDO 2015, en San Diego, California ${ }^{(30)}$. Otros centros, como el Gustav Roussy, están realizando estudios con sunitinib y axatinib para esta enfermedad. 


\section{Referencias}

1. Baudin E, Habra MA, Deschamps F, Cote G, Dumont F, Cabanillas M, et al Therapy of endocrine disease: treatment of malignant pheochromocytoma and paraganglioma. Eur J Endocrinol. 2014;171(3):R111-22.

2. Ayala-Ramirez M, Feng L, Johnson MM, Ejaz S, Habra MA, Rich T, et al. Clinical risk factors for malignancy and overall survival in patients with pheochromocytomas and sympathetic paragangliomas: primary tumor size and primary tumor location as prognostic indicators. J Clin Endocrinol Metab. 2011;96(3):717-25.

3. Pinzon-Tovar A, Diaz EM, Motta OY, Castro M. Nuevos casos de feocromocitoma en el Hospital Universitario de Neiva Revista Colombiana de Endocrinología, Diabetes y Metabolismo. 2015;2(3):63-70.

4. Dahia PL. Pheochromocytoma and paraganglioma pathogenesis: learning from genetic heterogeneity. Nat Rev Cancer. 2014;14(2):108-19.

5. L P. Das Ganglioma embryonale sympathicum (Sympathoma embryonale). Berl Klin Wschr 1912;49:16-22

6. Manger WM. An overview of pheochromocytoma: history, current concepts, vagaries, and diagnostic challenges. Ann N Y Acad Sci. 2006;1073:1-20.

7. F F. Ein Fall von doppelseitigem, völlig latent verlaufenen Nebennierentumor und gleichzeitiger Nephritis mit Veränderungen am Circulationsapparat und Retinitis. Arch Pathol Anat Physiol Klin Med 1886;103:244-63

8. Classics in oncology. A case of bilateral completely latent adrenal tumor and concurrent nephritis with changes in the circulatory system and retinitis: Felix Fränkel, 1886. CA Cancer J Clin. 1984;34(2):93-106.

9. Cotesta D, Petramala L, Serra V, Pergolini M, Crescenzi E, Zinnamosca L, et al. Clinical experience with pheochromocytoma in a single centre over 16 years. High Blood Press Cardiovasc Prev. 2009;16(4):183-93.

10. Zelinka T, Petrák O, Turková H, Holaj R, Strauch B, Kršek M, et al. High incidence of cardiovascular complications in pheochromocytoma. Horm Metab Res. 2012;44(5):379-84.

11. Thosani S, Ayala-Ramirez M, Roman-Gonzalez A, Zhou S, Thosani N, Bisanz A, et al. Constipation: An Overlooked, Unmanaged Symptom of Patients with Pheochromocytoma and Sympathetic Paraganglioma. Eur J Endocrinol. 2015.

12. Bravo EL. Pheochromocytoma: new concepts and future trends. Kidney Int 1991;40(3):544-56.

13. Mishra AK, Agarwal G, Kapoor A, Agarwal A, Bhatia E, Mishra SK. Catecholamine cardiomyopathy in bilateral malignant pheochromocytoma: successful reversal after surgery. Int J Cardiol. 2000;76(1):89-90.

14. Coupez E, Eschalier R, Pereira B, Pierrard R, Souteyrand G, Clerfond G, et al. A single pathophysiological pathway in Takotsubo cardiomyopathy: Catecholaminergic stress. Arch Cardiovasc Dis. 2014;107(4):245-52.

15. Agarwal G, Mishra AK, Kapoor A, Agarwal A, Bhatia E, Mishra SK. Reversal of catecholamine induced cardiomyopathy in a patient with bilateral malignant pheochromocytoma. J Assoc Physicians India. 2001;49:1193-6.
16. Kassim TA, Clarke DD, Mai VQ, Clyde PW, Mohamed Shakir KM. Catecholamine-induced cardiomyopathy. Endocr Pract. 2008;14(9):1137-49.

17. Sibal L, Jovanovic A, Agarwal SC, Peaston RT, James RA, Lennard TW, et al Phaeochromocytomas presenting as acute crises after beta blockade therapy. Clin Endocrinol (Oxf). 2006;65(2):186-90.

18. Booth J. A short history of blood pressure measurement. Proc R Soc Med 1977;70(11):793-9.

19. Waguespack SG, Rich T, Grubbs E, Ying AK, Perrier ND, Ayala-Ramirez M et al. A current review of the etiology, diagnosis, and treatment of pediatric pheochromocytoma and paraganglioma. J Clin Endocrinol Metab. 2010;95(5):2023-37.

20. Neumann HP, Vortmeyer A, Schmidt D, Werner M, Erlic Z, Cascon A, et al. Evidence of MEN-2 in the original description of classic pheochromocytoma. N Engl J Med. 2007;357(13):1311-5.

21. Favier J, Amar L, Gimenez-Roqueplo AP. Paraganglioma and phaeochromocytoma: from genetics to personalized medicine. Nat Rev Endocrinol. 2015;11(2):101-11.

22. Baysal BE, Ferrell RE, Willett-Brozick JE, Lawrence EC, Myssiorek D, Bosch A, et al. Mutations in SDHD, a mitochondrial complex II gene, in hereditary paraganglioma. Science. 2000;287(5454):848-51.

23. Qin Y, Yao L, King EE, Buddavarapu K, Lenci RE, Chocron ES, et al. Germline mutations in TMEM127 confer susceptibility to pheochromocytoma. Nat Genet. 2010;42(3):229-33.

24. Burnichon N, Cascón A, Schiavi F, Morales NP, Comino-Méndez I, Abermi $\mathrm{N}$, et al. MAX mutations cause hereditary and sporadic pheochromocytoma and paraganglioma. Clin Cancer Res. 2012;18(10):2828-37.

25. Comino-Méndez I, Gracia-Aznárez FJ, Schiavi F, Landa I, Leandro-García LJ, Letón R, et al. Exome sequencing identifies MAX mutations as a cause of hereditary pheochromocytoma. Nat Genet. 2011;43(7):663-7.

26. Castro-Vega LJ, Buffet A, De Cubas AA, Cascón A, Menara M, Khalifa E, et al. Germline mutations in FH confer predisposition to malignant pheochromocytomas and paragangliomas. Hum Mol Genet. 2014;23(9):2440-6.

27. Comino-Méndez I, de Cubas AA, Bernal C, Álvarez-Escolá C, Sánchez-Malo C Ramírez-Tortosa CL, et al. Tumoral EPAS1 (HIF2A) mutations explain sporadic pheochromocytoma and paraganglioma in the absence of erythrocytosis. Hum Mol Genet. 2013;22(11):2169-76.

28. Cascón A, Comino-Méndez I, Currás-Freixes M, de Cubas AA, Contreras L, Richter S, et al. Whole-exome sequencing identifies MDH2 as a new familial paraganglioma gene. J Natl Cancer Inst. 2015;107(5).

29. DeLellis R, Lloyd R, Heitz P, C E. World Health Organization Classification of Tumours. Pathology and Genetics of Tumors of Endocrine Organs. Lyon: IARCS Press; 2004.

30. Jimenez C, Pryma DA, Sullivan DC, Schwarz JK, Noto RB, Stambler N, et al. Long Term Follow-up of a Pivotal Phase 2 Study of Ultratrace® Iobenguane I-131 (AZEDRA ${ }^{\mathrm{TM}}$ ) in Patients with Malignant Relapsed/Refractory Pheochromocytoma (Pheo)/Paraganglioma (Para). Endocrine Reviews 2015;36(2):0R24-6. 